\title{
V. The double nature of nabla
}

\section{Frank Lauren Hitchcock}

To cite this article: Frank Lauren Hitchcock (1909) V. The double nature of nabla, Philosophical Magazine Series 6, 17:97, 113-125, DOI: 10.1080/14786440108636582

To link to this article: http://dx.doi.org/10.1080/14786440108636582

$$
\text { 册 Published online: } 21 \text { Apr } 2009 .
$$

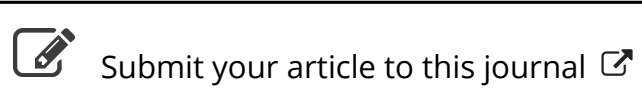

\footnotetext{
Џll Article views: 3
}

Q View related articles $\sqsubset$ 
a whole, while the velocity $\mathfrak{V}$ of compressional waves is given by

$$
\boldsymbol{\tau}^{2}=\frac{1}{8 \pi^{2} \Sigma\left(v / \Omega^{2}\right)} \cdot \quad . \quad . \quad .
$$

These expressions are given merely by way of illustration; they are of course far from being the most general, in the first place because of our assumption that all the vortices maly be treated as linear, and in the second place because it. has beeu supposed that the core of any one vortex either consists wholly of rotationally moving liquid or is wholly vacuous.

V. The Double Nature of Nabla. By Frank Lauren Нитсвсоск, Kenyon College, Gambier, Ohio, U.S.A.*

$1 \mathrm{THE}$ importance of the operator nabla in Physics must in 1 the nature of things continue to increase. The more wo come to regard a force between two material bodies as a manifestation of the properties of the intervening medium, the more any attempt to express facts in mathematical form leads to the use of the potential, and thence to nabla or some equivalent of nabla. Every actual distribution of force has a potential, which is sometimes a scalar, sometimes a vector, in general a quaternion, but in any case yields the force again when acted on by nabla. 'The same is true of every distribution of velocity in a fluid. Nabla might even be defined, from the point of view of a physicist, as that operation by which any quantity is derived from its potential $t$.

It is therefore of interest to inquire what are the most convenient methods of working with this operator and applying it to various kinds of functions. As is well known, nabla is a differentiator, which we may, if we wish, express in terms of three ordinary differentiations; and is also a vector, in a mathematical sense, obeying all the laws of vector algebra. We arrive at very direct methods of handling nabla as soon as we distinguish clearly these two properties, and take full advantage of the transformations to which they lead.

For, in the first place, nabla, in its capacity of differentiator, conforms to all the familiar rules for differentiation so long as we do not move it from its place relative to otler vectors. Vector multiplication is not commutative, but it is distributive, and, in its original form, it is associative, obeying, therefore,

* Communicated by the Author.

+ For a proof by the late Prof. C. J. Joly that every distribution of a quautity has a definite potential, see his appendix to Sir W. R. Hamilton's 'Elements of Quaternions,' 2nd ed., p. 451 .

Phil. Mag. S. 6. Vol. 17. No. 97. Jan. 1909. 
ordinary algebraic principles if the order of vectors in any term is not ehanged. The expressions thus obtaivable with nabla are noteworthy, both for their simplicity and their limitless variety; a few are given below, but are to be regarded as exemplifying the method rather than as constituting any attempt at completeness.

In the second place, as a vector, nabla satisfies the formulixe proved by Sir W. R. Hamilton for vectors and quaternions, so that by using these we may change the order of multipliers and bring nabla next to any desired operand. The variety of results obtainable by this method is even greater than by the other. The transformations which take the place of the commutative law of ordinary algebra are wonderfully manysided in their application.

2. As a significant example of these two ways of working with nabla, we may take the problem of applying nabla to the product of any two functions. Call these functions $q$ and $r$. They may be either scalars or vectors; in general they are quaternions. They are assumed to be finite and continuous in the portion of space considered. We then have immediately, by ordinary differentiation,

$$
\nabla\left(q r^{\prime}\right)=\nabla^{\prime} q^{\prime} r+\nabla^{\prime} q r^{\prime}, \ldots . . .
$$

where, on the left, nabla acts on both $q$ and $r$, but on the right is distributed, as indicated by the accents, accorciing to the familiar rule for the differentiation of a product; that is, in the first term on the right, nabla acts on $q$ but not on $r$, and in the last term it acts on $r$ but not on $q$; the order of multiplication is not changed in either term.

The first term on the right may, by the associative law of multiplication, be rewritten as $\nabla q, r$; this gives

$$
\nabla(q r)=\nabla q \cdot r+\nabla^{\prime} q r^{\prime}, . . . .
$$

a convenient equation for some purposes (to which I sball recur below). If, now, we wish to bring $\nabla$, in the last term, next to the operand $r$, we may do so by means of the formula

$$
\mathrm{K}\left(q_{1} q\right)=\mathrm{K} q \mathrm{~K} q_{1}
$$

true for any two quaternions $q_{1}$ and $q^{*}$. For this, by operating on both sides with $\mathrm{K}$, becomes

$$
q_{1} q=\mathbf{K}\left(\mathbf{K} q \mathbf{K} q_{1}\right) \text {, }
$$

because $K K=1 \uparrow$. This formula shows that we may invert the order of any two quaternions by introducing the symbol $\mathrm{K}$. With regard to notation, the parentheses on the right may well be omitted, for the first $\mathrm{K}$ obviously applies to all that follows it, otherwise the two adjacent K's would together be

* Hamilton, 'Elements of Quaternions,' 2nd ed., Art. 192.

+ Ibid. Art. 145 . 
equal to unity and would not be written down. Thus we have

$$
q_{1} q=\mathrm{KK}_{q} \mathrm{~K}_{q_{1}} \text {. }
$$

We may now write $\nabla$ in place of $q_{\mathrm{I}}$ in this formula, for $\nabla$ satisties all quaternion transtormations. Accordingly

$$
\begin{aligned}
\nabla q & =\mathrm{KK} q \mathrm{~K} \nabla \\
& =-\mathrm{KK}_{q} \nabla
\end{aligned}
$$

because the conjugate of a vector is its negative* This result is true whether $\nabla$ acts on $q$ or not, since it depends on the vectorial character of $\nabla$ and has nothing to do with differentiation. On substituting in (1 B) we may drop accents, with the usual understanding that an operator does not act to the left of itself unless accents are inserted to that effect ; then

$$
\nabla(q r)=\nabla q \cdot r-\mathrm{KK}_{q} \nabla \cdot r, . . .
$$
the period in the last term indicating that the first $\mathrm{K}$ acts only as far as $\nabla$.

3. Operators such as $K K q \nabla$, occurring in the last term of (1 C), are not always entirely comprehended at first sight, on account of their generality in comparison with ordinary differentiation. \& point of view from which all such operators may be directly worked with is indicated below (Art. 5). It will suffice here to give three illustrations of the methods by which we may, if we wish, introduce more elementary operators to suit different purposes.

First. snppose we wish to obtain, instead of $K \mathrm{~K} q \nabla$, a form which shall exhibit as obviously as possible its own relation to ordinary differentiation. We shall then naturally bear in mind that the notation connecting nabla with ordinary differentiation along a direction in space is $S \sigma \nabla$, where $\sigma$ is any vector along the direction of differentiation. In the present case this vector will be $V_{q}$; hence we shall so arrange the work as to bring in a term of the form $S V q \nabla$. Thus

$$
\begin{array}{rlrl}
\nabla q & =\nabla \mathrm{S} q+\nabla \mathrm{V}_{q}, & & \text { because } \mathrm{S}+\mathrm{V}=1, \\
& =\mathrm{S} q \cdot \nabla+\mathrm{K} \mathrm{V}_{q} \nabla, & & \text { by (3), } \\
& =\mathrm{S} q \cdot \nabla+(2 \mathrm{~S}-1) \mathrm{V}_{q} \nabla, & \text { because } \mathrm{K}=\mathrm{S}-\mathrm{V}=2 \mathrm{~S}-1, \\
& =\left(\mathrm{S} q-\mathrm{V}_{q}\right) \nabla+2 \mathrm{~S} \mathrm{~V}_{q} \nabla, & \text { identically, } \\
& =\mathrm{K}_{q} \cdot \nabla+\mathrm{S} \mathrm{V}_{q} \nabla, . & . & . \\
\end{array}
$$

and by substituting in ( $1 \mathrm{~B})$,

$$
\nabla(q r)=\nabla q \cdot r+\mathrm{K} q \cdot \nabla r+2 \mathrm{SV}_{q} \nabla \cdot r, .
$$

a useful expansion which is in the form we started to obtain;

* Ibid. Art. $1 \notin 4$. 
the only kind of differentiation implied in the first two terms on the right is the direct application of nabla; the other term implies ordinary differentiation along the direction $\mathrm{V} q$, or, if we preter to put $d x=f d \rho$, so that $f$ is the linear function obtained by differentiating $r$ completely, we may rewrite the last term as $-2 f^{\prime} \mathrm{V} q^{*}$.

Second, suppose we wish to obtain, instead of the operator $\mathrm{SV} q \nabla$, wlich is the scalar product of a vector and nabla, the corresponding vector operator $\mathrm{VV}, \nabla$, useful in the trunsformation of line and surface integrals + . Arranging the work so as to bring in such a term,

$$
\begin{aligned}
\nabla q & =\$ q \cdot \nabla+\mathrm{K} V q \nabla, \quad \text { as before, second line of }(4 \mathrm{~A}), \\
& =\mathrm{S} q \cdot \nabla+(1-2 \mathrm{~V}) \mathrm{V}_{q} \nabla, \text { because } \mathrm{K}=\mathrm{S}-\mathrm{V}=1-2 \mathrm{~V}, \\
& =(\mathrm{S} q+\mathrm{V} q) \nabla-2 \mathrm{~V}_{q} \nabla, \text { identically, } \\
& =q \nabla-2 \mathrm{~V} \mathrm{~V}_{q} \nabla, . . . . . . . . . . .
\end{aligned}
$$

and by substituting in (1 B),

$$
\nabla(q r)=\nabla q \cdot r+q \nabla r-2 V \nabla q \nabla \cdot r, \quad . \quad .
$$

which is in the form we started to obtain.

Third, suppose we wish to obtain that form of expansion which shall most clearly exhibit the quaternion equation as a generalization for three dimensions of the theory of ordinary complex quantities represented on a plane. We shall then keep nabla always at the left, and, instead of interchanging $\nabla$ and $q$, in the last term of $(1 \mathrm{~B})$, shall invert the order of the two quaternions $q$ and $r$; the steps are not unlike those in $(4 \mathrm{~B}):-$

$$
\begin{aligned}
& q r=(\mathrm{S} q+\mathrm{V} q)(\mathrm{S} r+\mathrm{V} r) \\
& =\mathrm{S}_{r} \mathrm{~S} q+\mathrm{V} r \cdot \mathrm{S} q+\mathrm{K} V_{r} \mathrm{~V}_{q} \text {, } \\
& =\mathrm{S} r \cdot q+\mathrm{V} r \cdot \mathrm{S} q+(1-2 \mathrm{~V}) \mathrm{V} r \mathrm{~V} q \text {, because } \mathrm{K}=1-2 \mathrm{~V} \text {, } \\
& =\mathrm{S} r \cdot q+\mathrm{V} r \cdot(\mathrm{S} q+\mathrm{V} q)-2 \mathrm{VVr} V q \text {, identically, } \\
& =r q-2 \mathrm{VVrV} q \text {, }
\end{aligned}
$$

scalars being commutative,

another formula for inverting two quaternions, giving by (1B)

$$
\nabla(q r)=\nabla q \cdot r+\nabla r \cdot q-2 \nabla^{\prime} V V r^{\prime} V_{q}, \quad .
$$

which is in the form we started to obtain, for if $q$ and $r$ are

* By putting two vectors for $q$ and $r$, and by operating with $\mathrm{S}$ or $\mathrm{V}$ eitber before or after such a substitution, we nay obtain a number of special cases very useful in practice. For another quite different method of obtaining them see Phil. Mag. [6] No. 18, June 1902, p. 580.

+ See, for instance, the physical applications in Tait's 'Quaternions,' Art. 497 et seq. 
in a fixed plane, their axes, or vector parts, are parallel to each other and to the vector parts of their differentials and the last term vanishes. The expansion then reduces to what it would be if $\nabla$ were the symbol of ordinary differentiation and $q$ and $r$ were scalars. That coplanar quaternions obey all the laws of ordinary algebra is well known.

These results may be extended. Thus for three quaternions, $q, r$, and $s$, by writing $q r$ for $q$ in $(1 \mathrm{~F})$, and $s$ for $r$,

$$
\begin{aligned}
\nabla(q r s) & =\nabla(q r) \cdot s+\nabla s \cdot q r-2 \nabla^{\prime} \mathrm{VV} s^{\prime} \mathrm{V} q r \\
& =\nabla q \cdot r s+\nabla r \cdot q s+\nabla s \cdot q r-2 \nabla^{\prime} V^{\prime} r^{\prime} \mathrm{V} q \cdot s \\
& -2 \nabla^{\prime} V^{\prime} s^{\prime} \mathrm{V} q r, .
\end{aligned}
$$

which may be transformed in a multiplicity of ways.

As a special case of (1 F) let $g=r$, then

$$
\nabla q^{2}=2 \nabla q \cdot q-2 \nabla^{\prime} \mathrm{VV} q^{\prime} \mathrm{V} q, . \quad \cdot .
$$

where the last term does not vanish unless $q$ and $d q$ are coplanar.

4. The result just obtained for the effect of nabla on the square of any quaternion suggests the question whether a simple general method exists for applying nabla to any function of a function. The answer is that, as has already been illustrated, the accents which we apply to $\nabla$ and to the operand may be treated in any way that the symbol $d$ of ordinary differentiation can be treated. Thus (1A) is true because

$$
d(q r)=d q \cdot r+q d r
$$

and we may similarly, in any differential identity, replace $d$ by an accent applied to the letter before which $d$ stands, and write $\nabla$ at the left of the whole : this is the first of the two characteristic properties of nabla mentioned in Art. 1. Therefore, if $\mathrm{F} q$ be any function of $q$ and we write

$$
d \mathrm{~F} q=f d q, . . \quad . \quad . \quad .
$$

where $f$ is the linear function obtained by differentiating $\mathrm{F} q$ completely, it at once follows that

$$
\nabla \mathrm{F} q=\nabla^{\prime} f q^{\prime}, \quad \text {. . . . . . . }
$$

which is the fundamental formula for applying nabla to a function of a function. The constituents of $f$ will in general contain unaccented $q$ as well as constants.

As an illustration, let us obtain (6) by this method. 
We have

$$
\begin{aligned}
d q^{2} & =d q \cdot q+q d q \\
& =2 d q \cdot q-2 \mathrm{VV} d q \mathrm{~V} q,
\end{aligned}
$$

by putting $r=d q$ in (3B), so that by (8)

$$
\nabla q^{2}=2 \nabla^{\prime} q^{\prime} q-2 \nabla^{\prime} V \mathrm{~V} q^{\prime} \mathrm{V} q
$$

which is the same as (6). The method, we repeat, consists in differentiating the given function, arranging the differential in any way that may suit our purpose, and then replacing $d q$ by an accented $q^{\prime}$, while $\nabla$ is written at the left of each term.

To obtain the important generalization of (6) where the exponent shall be any constant scalar whatever, we have therefore to differentiate $q^{n}$. To do so, we may think of the differentiation as conducted in two steps: first, as if the plane of $q$ were fixed, and $\mathrm{UVq}$ constant, second, as if the rest of the quaternion were constant. The sum of the two results will be the complete differential *. Call the first step $d_{0}$ and the second $d_{1}$, then in general

$$
d=d_{0}+d_{1} . \quad . \quad . \quad . \quad . \quad . \quad .
$$

The result of $d_{0}$ on $q^{n}$ will be the same in form as if $q$ were a scalar, since quaternions of constant plane act like scalars; that is

$$
d_{0} \eta^{n}=d_{0} q \cdot n q^{n-1}
$$

The result of $d_{1}$ implies no differentiation except $d U V q$, for $q^{n}$ is coplanar with $q$ itself, $\mathrm{UV}^{n}$ is the same as $\pm \mathrm{UV} q$, and we may write $\uparrow$

$$
q^{n}=\mathrm{S} q^{n} \pm \mathrm{TV} q^{n} . \mathrm{UV} q,
$$

whence, differentiating as if $\mathrm{UV} q$ alone were variable,

$$
d_{1} q^{n}= \pm \mathrm{TV} q^{n} . d \mathrm{UV} q, . . \quad .
$$

and by adding (10) and (11)

but by (9)

$$
d q^{n}=d_{0} q \cdot n q^{n-1} \pm \mathrm{TV} q^{n} \cdot d \mathrm{UV} q,
$$

$$
\begin{aligned}
d_{0} q & =d q-d_{1} q \\
& =d q-\mathrm{TV} q \cdot d \mathrm{UV} q .
\end{aligned}
$$

by the definition of $d_{1}$, which substituted in the preceding gives

$$
d q^{n}=d q \cdot n q^{n-1}+d \mathrm{UV} q \cdot\left( \pm \mathrm{TV} q^{n}-\mathrm{TV} q \cdot n q^{n-1}\right), .
$$

* Hamilton, Art. 329.

† The double sign is of course ambiguous in appearance only. We may, if we prefer, write $+\frac{\mathrm{V} q^{n}}{\mathrm{UV} q}$ instead of $\pm \mathrm{TV} q^{n}$ in equations (11)-(14). 
and as the differentials stand at the left of the terms we have immediately, by (8),

$$
\nabla q^{n}=\nabla q \cdot n q^{n-1}+\nabla \mathrm{UV} q \cdot\left( \pm \mathrm{TV} q^{n}-\mathrm{TV} q \cdot n q^{n-1}\right) \text {. }
$$

There is nothing in the reasoning employed which will not apply equally well if, instead of $q^{n}$, we take any other function of $q$ containing neither quaternion constants nor any of the selective symbols $\mathrm{S}, \mathrm{V}, \& \mathrm{c}$. ; in other words, if the functional form be any of those contemplated in the Newtonian, as distinguished from the Hamiltonian, differential calculus. For if we do not introduce any vector except $\mathrm{V} q$ into the functional operation, the result can contain no other vector, or scalar multiples, and the vector prart, axis, and plane of the function will be parallel to those of $q$ itself. All such functions of a single quaternion $q$ must therefore be commutative with one another. 'They wili also be commutative with the result of $d_{0}$ upon them, since that operator also can yield no other vector except scalar multiples of $U V q$.

It will be convenient to call any such function, agreeing in form with those treated in the usual text-books on differentiation, or, better, any function which we can construct by the employment of scalars only, an ordinary function. For example, $\sin (n q)$ is an ordinary function of the quaternion $q$ if $n$ is a scalar, but not if $n$ is a vector or a quaternion.

It will also be convenient to represent such a function by the symbol $Q$, and to write $D Q$ for the result of differentiating it with regard to $q$ as if $q$ were a scalar. Thus if $Q$ be any ordinary function of $q$ we shall always have

$$
\begin{aligned}
d \mathrm{Q} & =d_{0} \mathrm{Q}+d_{1} \mathrm{Q} \\
& =a_{0} q \cdot \mathrm{DQ} \pm \mathrm{TVQ} \cdot d \mathrm{UV} q \\
& =d q \cdot \mathrm{DQ}+d \mathrm{UV} q \cdot( \pm \mathrm{TVQ}-\mathrm{TV} q \cdot \mathrm{DQ})
\end{aligned}
$$

of which (14) is evidently a particular case*. We note that the only differentiation to be performed upon $Q$ is that denoted by $D$, namely ordinary differentiation as if $q$ were a scalar. 'The differentials $d q$ and $d U V q$ pertain to $q$ only, depend therefore on the distribution of $q$ with respect to the variables of which it may be a function, and have nothing to do with the relation of $Q$ to $q$. Thus we may, by (15), at once write down the differentials of any of the functions which Hamilton has defined in the Elements, or any which we might ourselves define in case we use forms not treated by him (e.g. elliptic

* We may write $+\frac{\mathrm{VQ}}{\mathrm{UVq}}$ instead of $\pm T V Q$ in (15). A similar remark applies to equations (16)-(26). 
functions), provided such forms are what were just above called ordinary functions. For example,

$$
d e^{q}=d q \cdot e^{q}+d \mathrm{UV}_{q} \cdot\left( \pm \mathrm{TV}_{\epsilon}^{q}-\mathrm{TV}_{q} \cdot e^{q}\right), .
$$

and similarly

$$
d \sin q=d q \cdot \cos q+d \mathrm{UV} q \cdot( \pm \mathrm{TV} \sin q-\mathrm{TV} q \cdot \cos q),
$$

and so on. If the plane of $q$ is fixed, so that $U V q$ is constant, it is evident that the right side of (15), or of any special case of it, reduces to its first term. The presence of the term, or terms, in $d U V q$ is due to the fact that the quaternion expansion is valid for space of three dimensions, so that the ordinary formulæ have to be generalized. This is an illustration of the truth referred to in Art. 3 that the Quaternion Calculus is an extension of the system, due to Bueé, Argand, and others, by which a complex quantity is represented on a plane. It is the only such extension which preserves both the distributive and the associative laws.

In (15), or in any special case of it, we may replace $d$ by $\nabla$, thus,

$$
\nabla \mathrm{Q}=\nabla q \cdot \mathrm{DQ}+\nabla \mathrm{UV} q \cdot( \pm \mathrm{TVQ}-\mathrm{TV} q \cdot \mathrm{DQ}),
$$

$Q$ being any ordinary algebraic or transcendental function of the quaternion $q$. As an instance,

$$
\nabla \log q=\nabla q \cdot q^{-1}+\nabla \mathrm{UV} q \cdot\left( \pm \mathrm{TV} \log q-\mathrm{TV} q \cdot q^{-1}\right)
$$

TV $\log q$ as defined by Hamilton is the angle, or the amplitude, of $q$.

More generally, if $\mathrm{FQ}$ be any quaternionic function of $\mathrm{Q}$, we have

$$
d \mathrm{FQ}=f d \mathrm{Q},
$$

where $f$ is the linear function obtained by differentiating $\mathbf{Q}$. Then by (15)

$$
d \mathrm{FQ}=f\left[d q \cdot \mathrm{DQ}+d \mathrm{UV}_{q} \cdot( \pm \mathrm{TVQ}-\mathrm{TV} q \cdot \mathrm{DQ})\right]
$$
and by (8)

$$
\nabla \mathrm{FQ}=\nabla^{\prime} f\left[q^{\prime} \mathrm{DQ}+\mathrm{UV} q^{\prime} \cdot( \pm \mathrm{TVQ}-\mathrm{TV} q \cdot \mathrm{DQ})\right]
$$

As an example under the last two results, we have, if $a$ and $b$ are any two constant quaternions,

$d(a \sin b q)=a b d q \cdot \cos b q+a d \mathrm{UV} b q \cdot( \pm \mathrm{TV} \sin b q-\mathrm{TV} b q \cdot \cos b q)$,

obtained from (20) by putting $a$ for $f$ and $b q$ for $q$. And by (21),

$\nabla^{\prime} a \sin b q^{\prime}=\nabla^{\prime} a b q^{\prime} \cos b q+\nabla^{\prime} a \mathrm{UVV} b q^{\prime} \cdot( \pm \mathrm{TV} \sin b q-\mathrm{TV} b q \cdot \cos b q)$ 
In the most general case of a function of a single quaternion $q$, we may have under $F$ several ordinary functions; call them $Q_{1}, Q_{2}$, \&c., to which will correspond $f_{1}, f_{2}, \ldots$, giving

$$
d \mathrm{~F}\left(\mathrm{Q}_{1}, \mathrm{Q}_{2}, \ldots\right)=f_{1} d \mathrm{Q}_{1}+f_{2} d \mathrm{Q}_{2}+\ldots ; .
$$

each of the ' $f$ 's being obtained by differentiating the given function as if only the corresponding $Q$ were variable *. On the right we may substitute the value of each of the differentials from (15).

If we have a function of several quaternions, $q, r, \& c$. , the formulæ (15) and (24) will still serve, but the Q's and the $f$ 's will contain any or all of these quaternions. When we change $d$ for $\nabla$, we shall accent only the variables which we write in place of the differentials. As an example,

$$
\begin{aligned}
& d \log q r=d(q r) \cdot(q r)^{-1}+d \mathrm{UVV} q r \cdot\left( \pm \mathrm{TV} \log q r-\mathrm{TV} q r \cdot r^{-1} q^{-1}\right), . \\
& \quad \text { and changing to } \nabla,
\end{aligned}
$$

$\nabla \log q r=\nabla(q r) \cdot(q r)^{-1}+\nabla^{\prime} U V q^{\prime} r^{\prime} \cdot\left( \pm \mathrm{TV} \log q r-\mathrm{TV} q r \cdot r^{-1} q^{-1}\right)$,

in which we may, if we wish, transform UVqr by the known methods for differentiating a unit vector.

Most of the formulæ of the present section contain, on the right, the differential, or the nabla, of some unit vector. The differential of any unit vector is always at right angles to the unit vector itself $\uparrow$. If the unit vector is normal to a family of surfaces, it is also at right angles to its vector nabla ; in which case the unit vector, its nabla, and the new vector obtained by differentiating the unit vector along its own direction, form a rectangular system $\ddagger$. Thus the term by which the differential, or the nabla, of a quaternion function differs in form from that of a scalar may receive a geometrical interpretation.

5. In Art. 2, above, we had terms containing the operators $\mathrm{SV} q \nabla, \mathrm{VV} q \nabla$, and $\mathrm{KK} q \nabla$; and the terms on the right of (23) can, by the methods of Art. 2, be thrown into similar form. These operators are special cases of the more general expression $f \nabla$, where $f$ stands for any linear function; such functions of nabla are of great use, for example in hydrodynamics, and in the study of an anisotropic medium $\$$.

- Hamilton, Art. 329.

$\dagger$ Hamilton, Art. 335.

$\ddagger$ For the proof of this, with other properties of the rectaugular system, see Phil. May. [6] vol. iii. p. 581, and iv. p. 187.

\$ Maxwell, 'Electricity and Magnetism,' 3rd ed., Art. 101, $h$. 
Since in Art. 4 use was made of nabla in its capacity as differentiator alone, we may, wherever nabla occurs in any of the formulæ of that article, write a linear function of nabla instead of nabla itself. Thus instead of (8) we may write

$$
f \nabla . \mathrm{F} q=f \nabla^{\prime} f_{1} q^{\prime}, \text {. . . . . }
$$

where $f_{1}$ is the linear function obtained by differentiating $\mathrm{F} q$, but $f$ is any linear function whatever. The operator $f \nabla$, since it includes scalar functions of $\nabla$, includes ordinary differentiation as a very special case. Therefore (27) virtually includes all the results of the last article. The expansions (15) and (24) may be used whenever one or more ordinary algebraic or transcendental functions enter into $\mathrm{F} q$, which may be any function of $q$ whatever.

'The properties of linear functions afford many ways of transforming the right side of (27). For example, Hamilton showed that any linear function of a quaternion $q$ may be expressed as the sum of several terms of the form $t q s$, where $t$ and $s$ are quaternions*. Therefore the right of (27) may be expressed as the sum of several terms of the form $r \nabla^{\prime} s q^{\prime} t$, where $r, s$, and $t$ are quaternion functions which will in general contain the variable $q$ without accent. Each of these terms may then be transformed so as to bring $\nabla$ next to the operand, as in ( $1 \mathrm{D}$ ) and ( $1 \mathrm{E}$ ), or if we prefer, so as to bring $\nabla$ to the left of the whole, as in (1F). Such methods are still possible even when the expression to be transformed contains the selective operators $\mathrm{S}, \mathrm{V}$, or $\mathrm{K}$, for if $\alpha$ and $\beta$ are any two vectors we might put $2 \mathrm{~S} \alpha \beta=\alpha \beta+\beta \alpha$, and $2 \mathrm{~V} \alpha \beta=\alpha \beta-\beta \alpha$; so that by expanding sufficiently we might always arrive at an expression free from these selective symbols. We have, however, direct methods for handling $\mathbf{S}$ and $\mathrm{V}$ too fully illustrated by Hamilton and Tait to need exemplification here. No matter what the form of the original term, we may always transform so as to bring $\nabla$ next to any desired factor of the term.

Another general method is to put $d q=f_{2} d \rho$, where $f_{2}$ is the linear function obtained by differentiating $q$. Instead of (27) we then have

$$
f^{\prime} \nabla . \mathrm{F} q=f \nabla^{\prime} f_{1} f_{2} \rho^{\prime} ; . \quad \cdot \quad . \quad . \quad .
$$

and since it is no longer necessary to separate $f_{1}$ and $f_{2}$ we may more briefly write

$$
f \nabla . \mathrm{F}_{q}=f \nabla^{\prime} f_{3} \boldsymbol{\rho}^{\prime}
$$

in which $f_{3}$ is the linear function obtained by differentiating

$$
\text { * 'Elements,' Art. } 364 .
$$


F $q$ as a function of $\rho$; or it is the product of $f_{1}$ and $f_{2}$. We may then, as in the last paragraph, arrive at a sum of terms of the form $r \nabla^{\prime} s \rho^{\prime} t$, but by (4 B)

$$
\nabla s=s \nabla-2 \mathrm{VV} s \nabla
$$

whence we bring $\nabla$ next to $\rho$ and have a sum of expressions of the form

$$
r s \nabla \rho \cdot t-2 r \mathrm{VV} s \nabla \cdot \rho \cdot t,
$$

and because $\nabla \rho=-3$ and $V V s \nabla \cdot \rho=-2 V s^{*}$, we obtain a sum of expressions of the form

$$
-3 r s t+4 r V s \cdot t
$$

and may write in general

$$
f \nabla \cdot \mathrm{F} g=\mathbf{\Sigma}(-3 r s t+4 r \mathrm{~V} s . t) . .
$$

in which $r, s$, and $t$ are the quaternions obtained by expanding the right side of (28) or (29). The result (30), apart from practical utility, has a certain interest by showing (what is well known), that we may find the nabla of any quantity (and the more general $f \nabla$ ), withont ever introducing axes of reference, whether rectangular or not; and by means of one differentiation, not three,-a fact which might be commended to the attention of those who think that quaternions are mere abbreviations for Cartesian expressions.

We may, however, if we choose, introduce $i, j$, and $k$ into the work. The original definition of $\nabla$ gives, by (29),

$$
f \nabla \cdot \mathbf{F} q=f i \cdot f_{3} i+f j \cdot f_{3} j+f i \cdot f_{3} k, . \quad .
$$

and the right side is an invariant of the two linear functions $f$ and $f_{3}$. The late C. J. Joly showed how such invariants may be calculated, given the functions $f$ and $f_{3} \dagger$.

A fourth mothod, sometimes the shortest in practice, is to write the expression $f \nabla^{\prime} \cdot f_{3} \rho^{\prime}$ as a sum of terms in whatever way may be most convenient, then calculate the invariants of each term separately, and add. The advantage of this method is that it is always possible to express a linear function as the sum of a small number of standard forms, whose invariants are easily remembered; as, for example, that used above, $\mathrm{VV} s \nabla \cdot \rho=-2 \mathrm{~V} s$, or the very common form $\nabla^{\prime} \beta S \alpha \rho^{\prime}=-\alpha \beta$.

To sum up, we may find the result of applying any linear function of nabla to any given function by performing a

* 'Tait, 'Quaternions,' Art. 145-6.

† Appendix to Hamilton's 'Elements,' 2 nd ed., chapter v.; and Trans. R. I. A. xxx. p. 709. 
single differentiation upon the given function, and transforming to suit the purpose in view. The fundamental formula for the work is (27), which contains the others.

6 . A full discussion of the general case, when $f \nabla$ is any function of $\nabla$, not necessarily linear, would exceed the limits of this paper. A few illustrations of the meaning and use of such operators must suffice.

The simplest function of the second order in nabla is the familiar $\nabla^{2}$. Another is $\mathrm{S} \sigma \nabla \mathrm{S} \nabla \nabla$, which implies successive differentiation of the operand along two different directions $\sigma$ and $\tau$. The most general scalar function homogeneous and of the second order in nabla is of the form $\Sigma \nabla \phi \nabla$, where $\phi$ is any self-conjugate linear vector function.

Vector functions of nabla depend on the properties of vector functions of a vector. No general theory has been worked out except for linear functions. In a future paper I hope to show that a vector function homogeneous of the second degree in a vector $\lambda$ may be put in the form

$$
\mathrm{V} \phi \lambda \theta \lambda+\lambda \mathrm{S} \alpha \lambda
$$

where $\phi$ and $\theta$ are linear vector functions and $\alpha$ is a vector. Then the most general vector function homogeneous of the second order in nabla would be of the same form, writing $\nabla$ for $\lambda$.

We may, however, write down at once equations like (27) for any differential operators whatever. Thus if $d^{\prime}$ and $d^{\prime \prime}$ are symbols of two independent differentiations, we shall have

$$
d^{\prime} d^{\prime \prime} \mathrm{F} q=f_{1}\left(d^{\prime} q, d^{\prime \prime} q\right)
$$

in which $f_{1}$ will be a function linear in each of the two differentials. Then if $f\left(\nabla^{\prime}, \nabla^{\prime \prime}\right)$ is any operator homogeneous and of the second order in nabla, the differential nature of nablia onables us to write

$$
f\left(\nabla^{\prime}, \nabla^{\prime \prime}\right) . \mathrm{F} q=f\left(\nabla^{\prime}, \nabla^{\prime \prime}\right) \cdot f_{1}\left(q^{\prime}, q^{\prime \prime}\right), . .
$$

in which $\nabla^{\prime}$ acts on $q^{\prime}$ and $\nabla^{\prime \prime}$ on $q^{\prime \prime}$. Since $d^{\prime}$ and $d^{\prime \prime}$ are independent, $f_{1}$ is symmetrical in its two operands, a property not in general possessed by $f$ in the two nablas. One or both nablas will usually act on the constituents of $f$.

7. The commutation of these differential operators is a matter of importance both in Physics and in Mathematics. Tait gives a proof of the celebrated theorem that rortex motion cannot originate in a frictionless medium, by commuting $\frac{\delta}{\delta t}$ with $\mathrm{V} \nabla^{*}$. Commuting two ordinary differentiations along different directions gives rise to the so-called "Klammerausdruck," due to Jacobi, and important * 'Quaternions,' 3rd ed., Art. 313. 
in Lie's Theory of Transformation Groups. If we have any two linear functions of $\nabla$, which we may call $f \nabla$ and $g \nabla$, they will be amenable to commutation by $(3 \mathrm{~B})$ whether or not either of them acts on the constituents of the other, but usually each will act on all that follows it; if so, we have, by Hamilton, Art. 329,

$$
f^{\prime} \nabla \cdot g \nabla \cdot q=f^{\prime} \nabla^{\prime} \cdot g^{\prime} \nabla \cdot q+f \nabla^{\prime} \cdot g \nabla^{\prime} \cdot q^{\prime}, . .
$$

and with the operators in inverted order,

$$
g \nabla \cdot f^{\prime} \nabla \cdot g=g \nabla^{\prime} \cdot f^{\prime} \nabla \cdot q+g \nabla^{\prime} \cdot f \nabla^{\prime} \cdot q^{\prime}, . .
$$

in both of which identities $\nabla$, if without accent, acts on all that follows it in the same term, but, if accented, acts only on the accented factor. The terms last on the right of (34) and (35) are amenable to commutation by (3 B), whence the difference of these two terms is $2 \mathrm{VV} f^{\prime} \nabla^{\prime} \mathrm{V} g \nabla^{\prime} \cdot q^{\prime}$ and by subtracting (35) from (34),

$$
\begin{aligned}
(f \nabla \cdot g \nabla \cdot q-g \nabla \cdot f \nabla \cdot q)= & f \nabla^{\prime} \cdot g^{\prime} \nabla \cdot q-g \nabla^{\prime} \cdot f^{\prime} \nabla \cdot q \\
& +2 \mathrm{~V} \vee f \nabla^{\prime} \nabla_{g} \nabla^{\prime} \cdot q^{\prime} ; .
\end{aligned}
$$

in the last term both $\nabla$ 's act on $q$ only, but they both act on it, making this term one of the second order. The last term vanishes $(a)$ if one or both of the functions $f$ and $g$ is only a scalar function, or $(b)$ if the vector parts of these two functions are parallel. The "Klammerausdruck" of Jacobi comes, of course, under (a). We might, by using three operators, work out an analogue of the "Jacobian identity."

8. Finally, we may, in the general formulæ (33) and (36), and in the whole of Art. 4 , write $\nabla+\frac{d}{d t}$ instead of $\nabla$, where $\frac{d}{d t}$ means taking the partial derivative with regard to some scalar variable, usually the time $t$. For these results are consequences of the differential character of nabla, and are equally valid if we put the quaternion $\nabla+\frac{d}{d t}$ for the vector $\nabla$. If we do so, we shall best follow Prof. Kimura * in regarding $\nabla+\frac{d}{d t}$ as an extended nabla, and represent it by a single symbol. Its quaternion character makes it amenable to any of the processes used in Art. 3 and Art. 5 of this paper, and the results will differ only slightly from those there obtained. Naturally, in using the extended operator, we imply that the operand is variable both in space and in time, or in some other scalar. 